\title{
A bottom-up approach for forecasting GDP in a data rich environment
}

\author{
Francisco Dias \\ Banco de Portugal
}

\author{
Maximiano Pinheiro \\ Banco de Portugal
}

\author{
António Rua \\ Banco de Portugal
}

July 2016

\begin{abstract}
In an increasingly data-rich environment, the use of factor models for forecasting purposes has gained prominence in the literature and among practitioners. In this article, we extend the work of Dias, Pinheiro and Rua (2015) by assessing the forecasting behaviour of factor models to predict several GDP components and investigate the performance of a bottomup approach to forecast Portuguese GDP growth. We find supporting evidence of the usefulness of factor models and noteworthy forecasting gains when conducting a bottomapproach drawing on the main aggregates of GDP. (JEL: C22, C53)
\end{abstract}

\section{Introduction}

$\mathrm{O}$

ver the past decades the stream of economic information available

to policymakers increased enormously owing to the widespread development of statistical systems. Naturally, economic agents and in particular international organizations and central banks, as a general practice, follow these large information sets when assessing the ongoing economic developments and designing policy responses. In such a data rich environment, tackling an information set which can comprise hundreds of time series raises methodological challenges in terms of econometric modelling.

In particular, the use of factor models for forecasting purposes has become an increasingly widespread tool to forecast macroeconomic variables in a data rich environment. See, for example, Stock and Watson $(1998,2002 a, b)$ and Giannone et al. (2008) for the United States, Marcellino et al. (2003) and Angelini et al. (2011) for the euro area, Artis et al. (2005) for the UK, Schumacher (2007, 2010, 2011) for Germany, Barhoumi et al. (2010) for France, de Winter (2011) and Reijer (2013) for the Netherlands, and for a cross-country study encompassing several European countries see Rünstler et al. (2009). 
Factor models are designed to reduce the overall size of the number of series in large datasets to a manageable scale so as to circumvent the issue of the curse of dimensionality. Basically, these models condense the informational content of large datasets in a set of few unobserved series, the common factors, which account for a sizeable fraction of the overall comovements amongst the entire set of series. Hence, these factors capture in a parsimonious way the main features of the dataset and are therefore included as regressors in forecasting models in place of the original variables.

However, the part of the information other than the one conveyed by the small set of estimated factors is not considered in the forecasting equation. Such a procedure may disregard potentially relevant information for the variable to be forecasted or the forecast horizon under consideration.

To overcome this potential caveat Dias et al. (2010) proposed an alternative procedure. In particular, a targeted diffusion index was suggested that takes on board not only all the factors of the database but also their information content for the variable being forecasted and the forecast horizon. This index consists of a double weighted average of all the factors of the dataset that take into account both the explanatory power of each factor for the variable to be forecasted as well as the relative importance of the factor in capturing the co-movements of the series in the database. In an application for the United Sates, such an approach proved superior to the standard factor model in forecasting several macroeconomic variables. Furthermore this method has also been recently applied by Dias et al. (2015) for Portugal to forecast GDP growth providing encouraging results.

Herein, we extend the work by Dias et al. (2015) and assess the usefulness of the above-mentioned factor models to forecast GDP components.

Typically, two key reasons are pointed out to motivate the importance of such an assessment. First, forecasting the disaggregated components enhances a more comprehensive understanding of the aggregate. In many institutions, such as in central banks, it is crucial to have a full picture of the underlying economic developments so as to enhance the formulation of useful economic policies.

Second, there is the traditional debate on whether one should forecast an aggregate variable directly or indirectly by aggregating the forecasts of its components. In particular, the accuracy of forecasting directly the aggregate is compared with the performance obtained via a bottom-up approach. Previous empirical work along this line includes Fair and Shiller (1990) for the United States GNP, Zellner and Tobias (2000) for GDP growth in industrialized countries, Marcellino et al. (2003) for several euro area aggregates, Hubrich (2005) and Duarte and Rua (2007) for inflation in the euro area and Portugal, Esteves (2013) for euro area GDP, among others.

From a theoretical standpoint, it has been argued that if the data generating process is known for all components then the forecast obtained by aggregation of the disaggregated forecasts dominates in terms of forecasting performance 
(see, for example, Rose (1977), Tiao and Guttman (1980), Kohn (1982) and Lütkepohl (1984). However, in practice, the data generating processes are not known and instead must be specified and estimated. In such a context, the superiority of the bottom-up approach is no longer assured (see Lütkepohl, 1984). Hence, it boils down to an empirical issue.

In this article, we evaluate the relative forecasting performance of factoraugmented models to predict each individual GDP component. Thereafter, drawing on the forecasts for the GDP components, we assess if pursuing a bottom-up approach can deliver forecasting gains when predicting GDP growth.

\section{Brief overview of factor models}

Factor models were initially developed in the late seventies and early eighties by Geweke (1977), Sargent and Sims (1977) and Geweke and Singleton (1981). However the empirical applications of these models until the early nighties were confined to a handful set of variables.

Stock and Watson $(1998,2002 a, b)$ and Forni et al. (2000, 2001, 2004, 2005) contributed to a large extent to this literature extending the possibility of applications of factor models to datasets comprising hundreds of series. In fact, James Stock and Mark Watson, in their seminal work, were the first to propose the use of the principal component method to estimate common factors in the presence of a large number of economic series. Due to its generality and overall simplicity, it became quite popular among forecasters. In particular, we will confine our exercise to the static factor framework. Note that the dynamic version of the factor model specification can be rewritten in an equivalent static factor formulation (see, for example, Stock and Watson, 2005). Moreover, for forecasting purposes, which is the focus of this article, such distinction is pretty much irrelevant as mentioned by Bai and $\mathrm{Ng}$ (2007).

In the static factor model the forecasting exercise comprises two stages. In the first step, which involves the estimation of the factors, the model specification assumes that each and every variable in the large dataset results as a sum of two components: the first which is a linear combination of a small set of latent unobserved static factors, which is common to all variables, and an idiosyncratic component which is specific to each variable. Under these circumstances it has been proposed to estimate the unobserved common factors relying on the principal components technique which is shown to provide a consistent estimator of the factor space under fairly general conditions.

The principal components are ordered according to their relative importance which reflects its ability to capture the common dynamics in the whole dataset. Typically, the few major top-ranked principal components capture a sizeable share of the comovements amongst the series in the dataset, 
and the relevant number of factors to be considered in the forecasting model in step two can be chosen relying on some information criteria (see, for example, Bai and Ng, 2002).

In the second step, the variable to be forecasted is projected on the set of factors obtained in the previous step (which corresponds to the socalled Diffusion Index (DI) model) and possibly on lags of the dependent variable (termed DI-AR model). In this setting, whichever variable is being forecasted the same set of factors is included in the forecasting equation, i.e., the dependent variable plays no role in the selection of the factors to be considered in the regression.

Hence, the entire set of lower ranked factors are discarded, irrespectively of their information content for the variable to be forecasted and the forecasting horizon, which may lead to a loss of potentially useful information. Under these circumstances, the standard modelling procedure may prove to be limited for forecasting purposes since it does not take into account neither the specific variable to be forecasted nor the forecast horizon in the selection of the factors to be included in the forecasting equation.

To overcome this shortfall, Dias et al. (2010) suggested a Targeted Diffusion Index (TDI) in place of the above mentioned set of main factors in the second step of the standard factor model approach. The TDI is a convex linear combination of all the factors of the dataset, where the weights attached to each factor reflect both the share of the total variance captured by the factor and its correlation with the variable to be predicted at the relevant forecast horizon. Thus, this procedure takes into account the entire set of factors combined in a single index using both the information conveyed by the relative importance of the factors in the spirit of Stock and Watson and the information content of each factor for the specific variable and horizon to be predicted.

This approach avoids dismissing potentially relevant information contained in the dataset and tries to obtain a better match between the available data and the variable to be predicted. Dias et al. (2010) show that such a modelling strategy improves substantially the forecasting performance vis-à-vis the DI model for several US macroeconomic variables while Dias et al. (2015) find encouraging results for forecasting GDP growth in Portugal.

\section{Forecasting GDP components}

\section{Preliminaries}

Concerning the data, we resort to the updated dataset compiled by Dias et al. (2015) for the Portuguese economy which comprises 126 series. It includes both hard and soft data covering business and consumers surveys (43 series), retail sales (4 series), industrial production (7 series), turnover in industries 
and services (20 series), employment, hours worked and wage indices in industries and services (24 series), tourism nights spent in Portugal (3 series), car sales ( 3 series), cement sales, vacancies and registered unemployment (5 series), energy consumption (3 series), nominal exports and imports of goods (10 series), real effective exchange rate, Portuguese stock market index and ATM/POS series. All series are seasonally adjusted and, with the exception of survey data, taken in logarithms. As usual, the series are then differenced.

Regarding GDP and its components, the series are available from the Portuguese National Statistics Office (INE) as from the first quarter of 1995 up to the fourth quarter of 2015 on a seasonally adjusted basis and in real terms. We focus on the corresponding quarter-on-quarter rates of change and restrict the analysis to nowcasting and forecasting one-quarter ahead horizon as the gains of using factor-augmented models are relatively negligible for more distant horizons (see also Dias et al., 2015).

The out-of-sample period runs from the first quarter of 2002 up to the fourth quarter of 2015. Such a long out-of-sample period allows us to put to test more thoroughly the relative performance of the competing models and allows us to consider a sub-sample analysis. In particular, we consider two sub-samples namely from 2002 Q1 up to 2007 Q4 and from 2008 Q1 up to 2015 Q4. The latter period corresponds to a challenging period by all standards as the Portuguese economy underwent marked macroeconomic changes since the latest economic and financial crisis.

As usual in this type of exercises, we consider as the benchmark a univariate autoregressive model with the lag order determined by standard BIC criteria in each round of the recursive expanding window exercise. In the case of the DI model, where the number of factors have to be determined exante, we consider four factors as discussed extensively in Dias et al. (2015).

We present the Mean Squared Forecast Error (MSFE) for the benchmark model and the relative MSFE for the competing models which is computed as the ratio between the MSFE of the competing model to the MSFE of the benchmark. Hence, a relative MSFE lower than one means that the competing model outperforms the benchmark whereas if it is higher than one it is the opposite. Finally, to assess the statistical significance of the forecasting gains relative to the benchmark we compute the Diebold and Mariano (1995) test (in the tables ${ }^{* * * * * *}$ denote statistical significance at 10,5 , and 1 per cent levels, respectively).

\section{Results}

\section{i) Private consumption}

Let us start with the analysis of the results for private consumption as a whole (see Table 1). For the entire out-of-sample period, both the DI and TDI models deliver statistically significant superior nowcasting performance. The gains 
vis-à-vis the benchmark are 26 per cent and 37 per cent, respectively. In terms of the behavior over the out-of-sample period, the gains are larger in the first part of the sample attaining 43 and 48 per cent, respectively. Although the gains are lower in the second part of the sample, they are still quite sizeable in particular in the case of the TDI model (33 per cent). Hence, the TDI model always presents the lowest relative MSFE for any sample period for nowcasting purposes.

\begin{tabular}{l|l|l|l|l|l|l}
\hline \hline Out-of-sample period & \multicolumn{2}{|c|}{ 2002Q1-2015Q4 } & \multicolumn{2}{|c|}{ 2002Q1-2007Q4 } & \multicolumn{2}{l}{ 2008Q1-2015Q4 } \\
\hline Forecast horizon & $h=0$ & $h=1$ & $h=0$ & $h=1$ & $h=0$ & $h=1$ \\
\hline & & & & & & \\
Total & 0.009 & 0.010 & 0.005 & 0.006 & 0.012 & 0.014 \\
AR model (MSFE) & $0.74^{*}$ & 1.03 & $0.57^{* *}$ & 1.48 & 0.80 & 0.89 \\
DI model (Relative MSFE) & $0.63^{* *}$ & 0.73 & $0.52^{*}$ & 0.95 & $0.67^{*}$ & 0.66 \\
TDI model (Relative MSFE) & & & & & & \\
& & & & & & \\
Durables & 0.389 & 0.380 & 0.225 & 0.236 & 0.511 & 0.488 \\
AR model (MSFE) & 0.73 & 0.92 & 0.86 & 1.13 & 0.68 & $0.84^{* * *}$ \\
DI model (Relative MSFE) & $0.61^{* *}$ & 0.88 & 0.64 & 1.39 & $0.60^{*}$ & $0.69^{* *}$ \\
TDI model (Relative MSFE) & & & & & & \\
& & & & & & \\
Non-durables & 0.005 & 0.005 & 0.004 & 0.004 & 0.006 & 0.007 \\
AR model (MSFE) & 0.88 & 1.16 & $0.42^{* * *}$ & 1.27 & 1.09 & 1.12 \\
DI model (Relative MSFE) & & & & & \\
TDI model (Relative MSFE) & 0.78 & 0.86 & $0.53^{* *}$ & 0.71 & 0.89 & 0.91 \\
\hline \hline
\end{tabular}

TABLE 1. Private consumption forecasting results

When forecasting one quarter-ahead, as expected, the gains are lower. In particular, the DI model does not outperform the benchmark while the TDI model presents a relative gain of 27 per cent, albeit not statistically significant. In this case, the forecasting performance turns out to be better in the second part of the sample for both models with the TDI model standing out.

We now turn to the components of private consumption namely durable and non-durables. In the case of nowcasting durables consumption, the gains obtained with DI and TDI models are similar to those recorded for private consumption as a whole. While the DI model seems to perform relatively better in the second part of the sample, the TDI model presents a stable performance across the sub-samples. For the one-quarter ahead horizon, the forecasting behavior worsens and the gains are substantially reduced. Nevertheless, the gains in the second part of the sample are statistically significant.

Regarding non-durables consumption, both DI and TDI models outperform the univariate benchmark for nowcasting. However, the gains are smaller than those reported above. In terms of its behavior over time, both models present a much better performance in the first part of the sample 
than in the second. For the one-quarter ahead horizon, the DI model does not outperform the benchmark whereas TDI does but not by much.

Overall, the TDI model delivers the best forecasting performance, both for the aggregate and any of its components. The gains are noteworthy when nowcasting whereas for the one quarter-ahead horizon the improvements are substantially reduced. Furthermore, these findings are relatively robust across sample periods.

\section{ii) Public consumption}

In the case of public consumption expenditures, we find that both the DI and TDI models perform better when augmented with autoregressive components determined by standard information criteria (denoted as DI-AR and TDI-AR, respectively). As one can see from Table 2, the DI-AR is not able to outperform the simple univariate autoregressive model when nowcasting or forecasting one-quarter ahead. Furthermore, this holds true for any out-of-sample period.

\begin{tabular}{l|l|l|l|l|l|l}
\hline \hline Out-of-sample period & \multicolumn{2}{|c|}{ 2002Q1-2015Q4 } & \multicolumn{2}{|c|}{ 2002Q1-2007Q4 } & \multicolumn{2}{l}{ 2008Q1-2015Q4 } \\
\hline Forecast horizon & $h=0$ & $h=1$ & $h=0$ & $h=1$ & $h=0$ & $h=1$ \\
\hline & & & & & & \\
AR model (MSFE) & 0.004 & 0.009 & 0.000 & 0.000 & 0.008 & 0.016 \\
DI-AR model (Relative MSFE) & 1.05 & 1.00 & 1.06 & 1.08 & 1.05 & 1.00 \\
TDI-AR model (Relative MSFE) & 0.96 & 0.82 & 0.89 & 0.82 & 0.96 & 0.82 \\
\hline \hline
\end{tabular}

TABLE 2. Public consumption forecasting results

In contrast, the TDI-AR model always presents a relative MSFE lower than one whatever the horizon or sample period. In particular, the relative gain is higher for forecasting one-quarter ahead (namely 18 per cent) than for nowcasting purposes. However, none of these improvements appear as statistically significant.

Hence, these results seem to support the view that it is hard to improve significantly over a simple autoregressive model for public consumption (see also Esteves, 2013). One should mention that quarterly public consumption in Portugal presents a relatively smooth profile as it results typically from the quarterly distribution of annual figures.

\section{iii) Investment}

The results concerning investment are reported in Table 3. Both the DI and TDI models present noteworthy gains when nowcasting investment as a whole namely 30 and 40 per cent, respectively. Similar sizeable gains are found across sample periods with the TDI model always performing better than the DI model. For the one-quarter ahead horizon, both the DI and TDI models deliver a gain around 10 per cent. Such an improvement reflects the behavior in the 
first part of the sample where the gains are quite large since in the second part of the sample the performance is close to the benchmark.

\begin{tabular}{|c|c|c|c|c|c|c|}
\hline \multirow{2}{*}{$\begin{array}{l}\text { Out-of-sample period } \\
\text { Forecast horizon }\end{array}$} & \multicolumn{2}{|c|}{ 2002Q1-2015Q4 } & \multicolumn{2}{|c|}{ 2002Q1-2007Q4 } & \multicolumn{2}{|c|}{ 2008Q1-2015Q4 } \\
\hline & $h=0$ & $h=1$ & $h=0$ & $h=1$ & $h=0$ & $h=1$ \\
\hline \multicolumn{7}{|l|}{ Total } \\
\hline AR model (MSFE) & 0.212 & 0.186 & 0.102 & 0.093 & 0.295 & 0.256 \\
\hline DI model (Relative MSFE) & $0.70^{* *}$ & 0.91 & 0.76 & 0.55 & $0.68^{*}$ & 1.01 \\
\hline TDI model (Relative MSFE) & $0.60^{* *}$ & 0.90 & 0.52 & $0.46^{*}$ & $0.62^{* *}$ & 1.02 \\
\hline \multicolumn{7}{|l|}{ Machinery and equipment } \\
\hline AR model (MSFE) & 0.853 & 0.996 & 0.190 & 0.193 & 1.351 & 1.598 \\
\hline DI model (Relative MSFE) & 0.86 & 0.77 & 1.01 & 1.21 & 0.85 & 0.73 \\
\hline TDI model (Relative MSFE) & 0.82 & 0.77 & 0.94 & 1.07 & 0.80 & 0.74 \\
\hline \multicolumn{7}{|l|}{ Transport equipment } \\
\hline AR model (MSFE) & 2.394 & 2.402 & 1.599 & 1.638 & 2.991 & 2.976 \\
\hline DI-AR model (Relative MSFE) & 0.89 & 0.91 & 1.03 & 1.07 & 0.83 & $0.84^{*}$ \\
\hline TDI-AR model (Relative MSFE) & 0.86 & 0.93 & 1.06 & 1.12 & $0.78^{*}$ & 0.86 \\
\hline \multicolumn{7}{|l|}{ Construction } \\
\hline AR model (MSFE) & 0.120 & 0.109 & 0.095 & 0.081 & 0.140 & 0.130 \\
\hline DI model (Relative MSFE) & 1.02 & 1.02 & 1.00 & 0.72 & 1.03 & 1.17 \\
\hline TDI model (Relative MSFE) & 0.87 & 1.09 & 0.58 & 0.82 & 1.02 & 1.21 \\
\hline \multicolumn{7}{|l|}{ Other } \\
\hline AR model (MSFE) & 4.614 & 5.227 & 4.890 & 5.493 & 4.406 & 5.027 \\
\hline DI model (Relative MSFE) & 0.99 & $0.79^{*}$ & 0.90 & $0.67^{*}$ & 1.07 & 0.88 \\
\hline TDI-AR model (Relative MSFE) & 1.02 & 0.86 & 1.13 & 0.78 & 0.93 & 0.93 \\
\hline
\end{tabular}

TABLE 3. Investment forecasting results

We also consider the various components of investment namely machinery and equipment, transport material, construction and other investment. Starting with machinery and equipment, both DI and TDI models improve on the univariate autoregressive model while delivering similar gains for both nowcasting and forecasting one-quarter ahead (around 20 per cent). Moreover, the gains are basically concentrated in the second part of the outof-sample period.

Regarding investment in transport equipment, the gains are more subdued (around 10 per cent) with both models presenting again a close relative performance for both horizons. The improvement is also more significant in the later part of the sample period.

Concerning construction, only the TDI model presents a gain for nowcasting as the DI model does not improve on the benchmark. For the one quarter-ahead horizon, none of the models deliver a gain for the outof-sample period as whole. In terms of sub-samples, both models perform 
relatively better in the first part of the sample although none of the gains are statistically significant.

Finally, for other investment, there are basically no gains to report when nowcasting while we find some improvement when forecasting one quarterahead, reflecting to a large extent the performance in the first part of the sample.

In sum, factor models tend to perform better, in most cases, than the benchmark. However, while for investment as a whole, the gains are clearly noteworthy with the TDI model standing out, the results for investment components are relatively disappointing. This seems to suggest that factor models can be potentially more useful when forecasting larger aggregates and not so much when one is interested in very specific components. This seems natural, as factor models tend to exploit the main commonalities in the data and therefore less appropriate to pinpoint narrow components.

\section{iv) Exports}

In Table 4 we report the results for aggregate exports as whole as well as for both exports of goods and services separately. Concerning total exports, we find large gains in both DI and TDI models when nowcasting and to a lesser extent for the one-quarter ahead horizon, with the TDI model performing slightly better than the DI model. For both horizons and in the case of the two models the gains are larger in the second part of the sample although not statistically significant as the improvement seems to be concentrated in a small number of observations.

\begin{tabular}{l|l|l|l|l|l|l}
\hline \hline Out-of-sample period & \multicolumn{2}{|c|}{ 2002Q1-2015Q4 } & \multicolumn{2}{|c|}{ 2002Q1-2007Q4 } & \multicolumn{2}{l}{ 2008Q1-2015Q4 } \\
\hline Forecast horizon & $h=0$ & $h=1$ & $h=0$ & $h=1$ & $h=0$ & $h=1$ \\
\hline & & & & & & \\
Total & 0.079 & 0.079 & 0.036 & 0.037 & 0.111 & 0.110 \\
AR model (MSFE) & 0.45 & 0.86 & 0.65 & 0.96 & 0.40 & 0.84 \\
DI model (Relative MSFE) & 0.42 & 0.80 & 0.68 & 1.00 & 0.36 & 0.76 \\
TDI model (Relative MSFE) & & & & & & \\
& & & & & & \\
Goods & 0.112 & 0.112 & 0.035 & 0.036 & 0.170 & 0.170 \\
AR model (MSFE) & 0.48 & 0.95 & 0.85 & 1.24 & 0.42 & 0.90 \\
DI model (Relative MSFE) & 0.42 & 0.86 & 0.84 & 1.11 & 0.35 & 0.82 \\
TDI model (Relative MSFE) & & & & & & \\
Services & & & & & & \\
AR model (MSFE) & 0.087 & 0.081 & 0.139 & 0.131 & 0.048 & 0.043 \\
DI model (Relative MSFE) & 1.07 & 1.01 & 0.75 & 0.98 & 1.77 & 1.08 \\
TDI model (Relative MSFE) & 0.98 & 1.29 & 0.73 & 1.09 & 1.51 & 1.75 \\
\hline \hline
\end{tabular}

TABLE 4. Exports forecasting results

In terms of components, there are noteworthy differences between goods and services. In the case of services, factor models do not seem to outperform 
the autoregressive benchmark. For exports of goods, the assessment is similar to the one reported above for exports as a whole albeit the gains are slightly lower. Such evidence seems to suggest that not much is gained from considering disaggregated exports.

\section{v) Imports}

We also assessed the relative performance of factor models to forecast imports (see Table 5). For imports as a whole, we find large and statistically significant gains when nowcasting, with the TDI model performing once again better than the DI model. The results are even stronger when one focus on the second part of the out-of-sample period. For the one-quarter ahead horizon, the gains are smaller and more visible in the first part of the sample.

\begin{tabular}{l|l|l|l|l|l|l}
\hline \hline Out-of-sample period & \multicolumn{2}{|c|}{ 2002Q1-2015Q4 } & \multicolumn{2}{|c|}{ 2002Q1-2007Q4 } & \multicolumn{2}{c}{ 2008Q1-2015Q4 } \\
\hline Forecast horizon & $\mathrm{h}=0$ & $\mathrm{~h}=1$ & $\mathrm{~h}=0$ & $\mathrm{~h}=1$ & $\mathrm{~h}=0$ & $\mathrm{~h}=1$ \\
\hline & & & & & & \\
Total & 0.100 & 0.095 & 0.049 & 0.054 & 0.138 & 0.126 \\
AR model (MSFE) & $0.56^{*}$ & 0.78 & 0.83 & 0.64 & $0.48^{*}$ & 0.82 \\
DI model (Relative MSFE) & & & & & \\
TDI model (Relative MSFE) & $0.48^{* *}$ & 0.77 & 0.65 & 0.69 & $0.43^{* *}$ & 0.79 \\
& & & & & & \\
Goods & & & & & & \\
AR model (MSFE) & 0.126 & 0.114 & 0.053 & 0.052 & 0.180 & 0.160 \\
DI model (Relative MSFE) & $0.50^{* *}$ & 0.81 & 0.71 & 0.70 & $0.46^{* *}$ & 0.84 \\
TDI model (Relative MSFE) & $0.45^{* *}$ & 0.78 & $0.56^{*}$ & 0.66 & $0.42^{* *}$ & 0.81 \\
& & & & & & \\
Services & & & & & & \\
AR model (MSFE) & 0.154 & 0.156 & 0.165 & 0.177 & 0.147 & 0.140 \\
DI model (Relative MSFE) & 1.21 & 1.00 & 1.19 & 1.14 & 1.23 & 0.87 \\
TDI model (Relative MSFE) & 1.15 & 1.13 & 1.08 & 1.33 & 1.20 & 0.95 \\
\hline \hline
\end{tabular}

TABLE 5. Imports forecasting results

In terms of components, likewise exports, we also find that there are only gains when one focuses on goods since factor models do not improve on the autoregressive model in the case of services. For imports of goods, the assessment is broadly similar to the one reported for imports as a whole.

\section{A bottom-up approach for GDP}

Given the forecasts for the several components of GDP, we now turn to the question of whether forecasting GDP growth can be improved via a bottomup approach or not. To assess if a bottom-up approach can deliver superior results than forecasting directly GDP growth, one has to pick a model to forecast it directly. Hence, we first evaluate the forecasting performance of 
the above considered models to forecast directly GDP growth, similarly to what has been done for each GDP component. In Table 6, we report the corresponding results.

\begin{tabular}{l|l|l|l|l|l|l}
\hline \hline Out-of-sample period & \multicolumn{2}{|c|}{ 2002Q1-2015Q4 } & \multicolumn{2}{|c|}{ 2002Q1-2007Q4 } & \multicolumn{2}{|c}{ 2008Q1-2015Q4 } \\
\hline Forecast horizon & $h=0$ & $h=1$ & $h=0$ & $h=1$ & $h=0$ & $h=1$ \\
\hline & & & & & & \\
AR model (MSFE) & 0.008 & 0.008 & 0.007 & 0.006 & 0.008 & 0.010 \\
DI model (Relative MSFE) & $0.49^{* *}$ & 1.00 & $0.42^{* *}$ & 1.01 & 0.53 & 1.00 \\
TDI model (Relative MSFE) & $0.37^{* * *}$ & 0.73 & $0.23^{* *}$ & $0.56^{* *}$ & $0.47^{*}$ & 0.80 \\
\hline \hline
\end{tabular}

TABLE 6. GDP forecasting results

As in Dias et al. (2015), we find that factor models outperform the benchmark with the TDI model standing out. ${ }^{1}$ For nowcasting, the gains achieved with the latter model are very large (63 per cent for the out-ofsample period as a whole) and statistically significant whatever the out-ofsample period considered. As expected, for the one-quarter ahead horizon the gains are smaller ( 27 per cent for the full out-of-sample period) and more pronounced in the first part of the sample. Hence, we will use the TDI model for forecasting directly GDP growth as the benchmark when evaluating the relative performance of the bottom-up approach for GDP.

In Table 7, we present the results for the bottom-up approach for GDP as well as for its main components. In particular, we first assess a bottom-up approach for each of the main aggregates of GDP, namely private consumption, investment, exports and imports. That is, we evaluate if forecasting each of the main GDP components directly is better than conducting the corresponding bottom-up approach. For instance, we analyze if forecasting directly private consumption is better than aggregating the forecasts of durables and non-durables using the corresponding national accounts weights. For each series we pick the overall best performing model based on the previous analysis, which we recall in the second column of Table 7 following the ordering of the discussion in the previous section.

1. These results do not correspond exactly to those reported in Dias et al. (2015) as quarterly national accounts have been revised by INE due to the adoption of ESA 2010 and the sample period herein considered has been extended. 


\begin{tabular}{|c|c|c|c|c|c|c|c|}
\hline \multirow{2}{*}{$\begin{array}{l}\text { Out-of-sample period } \\
\text { Forecast horizon }\end{array}$} & & \multicolumn{2}{|c|}{ 2002Q1-2015Q4 } & \multicolumn{2}{|c|}{ 2002Q1-2007Q4 } & \multicolumn{2}{|c|}{ 2008Q1-2015Q4 } \\
\hline & & $h=0$ & $h=1$ & $h=0$ & $h=1$ & $h=0$ & $h=1$ \\
\hline & Models & & & & & & \\
\hline \multicolumn{8}{|l|}{ Private consumption } \\
\hline Direct (MSFE) & 1) TDI & 0.006 & 0.007 & 0.003 & 0.005 & 0.008 & 0.009 \\
\hline Bottom-up (Relative MSFE) & 2) TDI+TDI & 1.03 & 1.01 & 1.14 & 1.05 & 1.00 & 0.99 \\
\hline \multicolumn{8}{|l|}{ Public consumption } \\
\hline Direct (MSFE) & 3) TDI-AR & 0.004 & 0.008 & 0.000 & 0.000 & 0.007 & 0.013 \\
\hline \multicolumn{8}{|l|}{ Investment } \\
\hline Direct (MSFE) & 4) TDI & 0.126 & 0.167 & 0.053 & 0.043 & 0.181 & 0.260 \\
\hline Bottom-up (Relative MSFE) & 5) TDI+TDI-AR+TDI+DI & 1.02 & 1.10 & 0.94 & 1.22 & 1.04 & 1.09 \\
\hline \multicolumn{8}{|l|}{ Exports } \\
\hline Direct (MSFE) & 6) TDI & 0.033 & 0.063 & 0.024 & 0.036 & 0.040 & 0.083 \\
\hline Bottom-up (Relative MSFE) & 7) $\mathrm{TDI}+\mathrm{AR}$ & 1.03 & 1.02 & 0.99 & 0.97 & 1.05 & 1.03 \\
\hline \multicolumn{8}{|l|}{ Imports } \\
\hline Direct (MSFE) & 8) TDI & 0.048 & 0.073 & 0.032 & 0.037 & 0.060 & 0.100 \\
\hline Bottom-up (Relative MSFE) & 9) $\mathrm{TDI}+\mathrm{AR}$ & 1.00 & 0.98 & 0.96 & $0.94^{* *}$ & 1.01 & 0.99 \\
\hline \multicolumn{8}{|l|}{ GDP } \\
\hline Direct (MSFE) & TDI & 0.003 & 0.006 & 0.002 & 0.003 & 0.004 & 0.008 \\
\hline Bottom-up with main aggregates (Relative MSFE) & 1)+3)+4)+6)+8) & $0.79^{*}$ & 0.97 & 0.84 & 1.39 & 0.78 & $0.85^{*}$ \\
\hline Bottom-up with detailed components (Relative MSFE) & 2)+3)+5)+7)+9) & 0.85 & 1.21 & 0.98 & 2.05 & 0.81 & 0.96 \\
\hline
\end{tabular}

TABLE 7. Forecasting via a bottom-up approach 


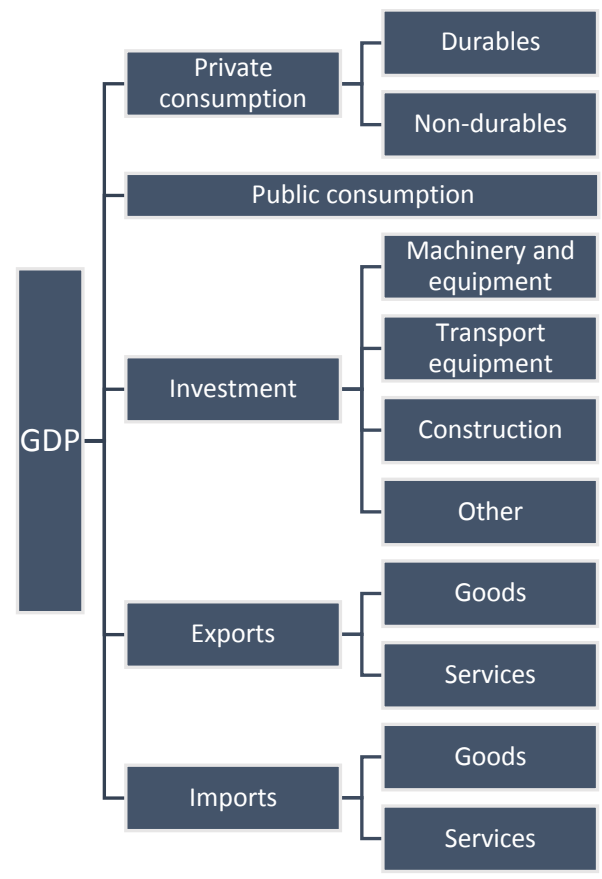

FIGURE 1: GDP disaggregation

We basically find that pursuing a bottom-up approach for each of the main GDP components does not improve the nowcasts neither the one-quarter ahead forecasts as the relative MSFE almost always exceeds one for the full out-of-sample period. This evidence seems to suggest that factor models are less useful when one focuses on relatively small components of GDP as they may be driven, to a large extent, by idiosyncratic forces. Naturally, given that factor models are designed to capture the main underlying common forces, the use of factor-augmented models tends to be less appropriate if one is interested in very narrow components of economic activity.

Regarding GDP, we consider two alternative levels of disaggregation for the bottom-up approach (see Figure 1). A level of disaggregation that considers the main aggregates of GDP (namely private consumption, public consumption, investment, exports and imports) and a higher level of disaggregation that uses more detailed GDP components (that is, consumption of durables, consumption of non-durables, public consumption, investment in machinery and equipment, transport equipment, construction, other investment, exports of goods, exports of services, imports of goods and imports of services). 
We find that the bottom-up approach improves substantially the nowcasts of GDP growth. ${ }^{2}$ In particular, the largest gains are attained when one considers the level of disaggregation that draws on the main aggregates of GDP. Such an approach delivers a statistically significant gain of 21 per cent over the best performing model for forecasting directly GDP growth. The gain is relatively stable across sub-samples.

For the one quarter-ahead horizon, the bottom-up approach that delivers the best results is once again the one that uses the main GDP components. Although it improves marginally vis-à-vis the direct forecast of GDP growth for the full out-of-sample period, there is a statistically significant gain of 15 per cent in the second part of the sample which is by all standards a very challenging period.

Hence, although a bottom-up approach does not seem to improve the forecasting performance in the case of the main aggregates of GDP, sizeable gains can be obtained when such an approach is pursued for GDP. This is particularly true when one considers a level of disaggregation based on the main GDP components. These results also reflect the fact that factoraugmented models are naturally more oriented to forecast broader aggregates of economic activity. ${ }^{3}$

\section{Conclusions}

Given the proved usefulness of factor-augmented models to forecast Portuguese GDP growth, we conducted a similar forecasting exercise to evaluate its performance in forecasting GDP components. As in the case of GDP, we find that factor models typically outperform the univariate autoregressive benchmark with the TDI model of Dias et al. (2010) standing out. Such an evidence reinforces the usefulness of the TDI approach in a broader context.

In particular, we find that the gains are larger when nowcasting and, as expected, decrease as one extends the forecast horizon. Moreover, we find that factor-augmented models are less useful when one forecasts relatively narrow components of GDP. In the presence of broadly based datasets, this seems a natural result as factor models tend to be more appropriate to capture the dynamics of broad GDP aggregates.

Drawing on the forecasts for the GDP components, we also assessed the forecasting behavior of the bottom-up approach vis-à-vis the direct approach.

2. We also considered the case where imports are modelled as a function of global demand weighted by the imported content (see Esteves et al., 2013) but it did not lead to an improvement in the forecasting performance.

3. In the appendix, we also report the corresponding results considering the Mean Absolute Forecast Error (MAFE) as in Dias et al. (2015). The findings are qualitatively similar. 
In this respect, we find significant gains when conducting a bottom-approach for GDP growth, in particular, when one considers a disaggregation level that draws on the main aggregates of GDP. 


\section{References}

Angelini, E., G. Camba-Mendez, D. Giannone, L. Reichlin, and G. Rünstler (2011). "Short-term forecasts of euro area GDP growth." Econometrics Journal, 14(1), C25-C44.

Artis, M., A. Banerjee, and M. Marcellino (2005). "Factor forecasts for the UK." Journal of Forecasting, 24, 279-298.

Bai, J. and S. Ng (2002). "Determining the number of factors in approximate factor models." Econometrica, 70, 191-221.

Bai, J. and S. Ng (2007). "Determining the number of primitive shocks in factor models." Journal of Business E Economic Statistics, 25, 52-60.

Barhoumi, K., O. Darné, and L. Ferrara (2010). "Are disaggregate data useful for factor analysis in forecasting French GDP?" Journal of Forecasting, 29(1-2), 132-144.

de Winter, J. (2011). “Forecasting GDP growth in times of crisis: private sector forecasts versus statistical models." DNB Working Papers 320, Netherlands Central Bank.

Dias, F., M. Pinheiro, and A. Rua (2010). "Forecasting using targeted diffusion indexes." Journal of Forecasting, 29(3), 341-352.

Dias, F., M. Pinheiro, and A. Rua (2015). "Forecasting Portuguese GDP with factor models: Pre- and post-crisis evidence." Economic Modelling, 44(C), 266-272.

Diebold, F. and R. Mariano (1995). “Comparing predictive accuracy." Journal of Business E Economic Statistics, 13, 253-263.

Duarte, C. and A. Rua (2007). "Forecasting inflation through a bottom-up approach: how bottom is bottom?" Economic Modelling, 24, 941-953.

Esteves, P. S. (2013). "Direct vs bottom-up approach when forecasting GDP: Reconciling literature results with institutional practice." Economic Modelling, 33, 416-420.

Esteves, P.S., F. Cardoso, and A. Rua (2013). "The import content of global demand in Portugal." Economic Bulletin, Autumn, 107-121, Banco de Portugal.

Fair, R. and J. Shiller (1990). "Comparing information in forecasts from econometric models." American Economic Review, 80, 375-389.

Forni, M., M. Hallin, M. Lippi, and L. Reichlin (2000). "The Generalized Dynamic-Factor Model: Identification And Estimation." The Review of Economics and Statistics, 82(4), 540-554.

Forni, M., M. Hallin, M. Lippi, and L. Reichlin (2001). "Coincident and Leading Indicators for the Euro Area." Economic Journal, 111(471), C62-85.

Forni, M., M. Hallin, M. Lippi, and L. Reichlin (2004). "The generalized dynamic factor model consistency and rates." Journal of Econometrics, 119(2), 231-255.

Forni, M., M. Hallin, M. Lippi, and L. Reichlin (2005). "The Generalized Dynamic Factor Model: One-Sided Estimation and Forecasting." Journal of 
the American Statistical Association, 100, 830-840.

Geweke, J. (1977). "The Dynamic Factor Analysis of Economic Time Series." In Latent variables in socio-economic models, edited by D. J. Aigner and A. S. Goldberger, chap. 19. North-Holland.

Geweke, J. and K. Singleton (1981). "Maximum likelihood 'confirmatory' factor analysis of economic time series." International Economic Review, 22(1), $37-54$.

Giannone, D., L. Reichlin, and D. Small (2008). "Nowcasting: The realtime informational content of macroeconomic data." Journal of Monetary Economics, 55(4), 665-676.

Hubrich, K. (2005). "Forecasting euro area inflation: does aggregating forecasts by HICP component improve forecast accuracy?" International Journal of Forecasting, 21, 119-136.

Kohn, R. (1982). "When is an aggregate of a time series efficiently forecast by its past?" Journal of Econometrics, 18, 337-349.

Lütkepohl, H. (1984). "Forecasting Contemporaneously Aggregated Vector ARMA Processes." Journal of Business \& Economic Statistics, 2(3), 201-214.

Marcellino, M., J. Stock, and M. Watson (2003). "Macroeconomic forecasting in the euro area: country specific versus euro wide information." European Economic Review, 47, 1-18.

Reijer, A. (2013). "Forecasting Dutch GDP and inflation using alternative factor model specifications based on large and small datasets." Empirical Economics, 44(2), 435-453.

Rose, D.E. (1977). "Forecasting aggregates of independent ARIMA processes." Journal of Econometrics, 5, 323-345.

Rünstler, G., K. Barhoumi, S. Benk, R. Cristadoro, A. Den Reijer, A. Jakaitiene, P. Jelonek, A. Rua, K. Ruth, and C. Van Nieuwenhuyze (2009). "Shortterm forecasting of GDP using large datasets: A pseudo real-time forecast evaluation exercise." Journal of Forecasting, 28(7), 595-611.

Sargent, T. J. and C. A. Sims (1977). "Business Cycle Modelling Without Pretending to Have too Much A-Priori Economic Theory." In New methods in business cycle research, edited by C. Sims et al. Federal Reserve Bank of Minneapolis.

Schumacher, C. (2007). "Forecasting German GDP using alternative factor models based on large datasets." Journal of Forecasting, 26(4), 271-302.

Schumacher, C. (2010). "Factor forecasting using international targeted predictors: The case of German GDP." Economics Letters, 107(2), 95-98.

Schumacher, C. (2011). "Forecasting with Factor Models Estimated on Large Datasets: A Review of the Recent Literature and Evidence for German GDP." Journal of Economics and Statistics, 231(1), 28-49.

Stock, J. and M. Watson (1998). "Diffusion Indexes." NBER Working Paper 6702, National Bureau of Economic Research.

Stock, J. and M. Watson (2002a). "Forecasting using principal components from a large number of predictors." Journal of the American Statistical 
Association, 97(460), 1167-1179.

Stock, J. and M. Watson (2002b). "Macroeconomic forecasting using diffusion indices." Journal of Business \& Economic Statistics, 20(2), 147-162.

Stock, J. and M. Watson (2005). "Implications of dynamic factor models for VAR analysis." NBER Working Paper 11467, National Bureau of Economic Research.

Tiao, G. C. and I. Guttman (1980). "Forecasting contemporal aggregates of multiple time series." Journal of Econometrics, 12, 219-230.

Zellner, A. and J. Tobias (2000). "A Note on aggregation, disaggregation and forecasting performance." Journal of Forecasting, 19(5), 457-469. 


\section{Appendix}

\begin{tabular}{|c|c|c|c|c|c|c|c|}
\hline \multirow{2}{*}{$\begin{array}{l}\text { Out-of-sample period } \\
\text { Forecast horizon }\end{array}$} & & \multicolumn{2}{|c|}{ 2002Q1-2015Q4 } & \multicolumn{2}{|c|}{ 2002Q1-2007Q4 } & \multicolumn{2}{|c|}{ 2008Q1-2015Q4 } \\
\hline & & $h=0$ & $h=1$ & $h=0$ & $h=1$ & $h=0$ & $h=1$ \\
\hline \multirow{2}{*}{\multicolumn{8}{|c|}{ Private consumption }} \\
\hline & & & & & & & \\
\hline Direct (MAFE) & 1) TDI & 0.55 & 0.64 & 0.38 & 0.57 & 0.69 & 0.69 \\
\hline Bottom-up (Relative MAFE) & 2) TDI+TDI & 1.01 & 1.01 & 1.06 & 1.03 & 0.99 & 0.99 \\
\hline \multicolumn{8}{|l|}{ Public consumption } \\
\hline Direct (MAFE) & 3) TDI-AR & 0.37 & 0.52 & 0.07 & 0.16 & 0.59 & 0.78 \\
\hline \multicolumn{8}{|l|}{ Investment } \\
\hline Direct (MAFE) & 4) TDI & 2.72 & 3.05 & 1.71 & 1.68 & 3.48 & 4.08 \\
\hline Bottom-up (Relative MAFE) & 5) TDI+TDI-AR+TDI+DI & 1.00 & 1.02 & 1.02 & 1.15 & 0.99 & 0.98 \\
\hline \multicolumn{8}{|l|}{ Exports } \\
\hline Direct (MAFE) & 6) TDI & 1.42 & 1.90 & 1.09 & 1.45 & 1.66 & 2.24 \\
\hline Bottom-up (Relative MAFE) & 7) $\mathrm{TDI}+\mathrm{AR}$ & 1.02 & 0.98 & 1.01 & 0.94 & 1.03 & 1.00 \\
\hline \multicolumn{8}{|l|}{ Imports } \\
\hline Direct (MAFE) & 8) TDI & 1.56 & 2.09 & 1.28 & 1.46 & 1.78 & 2.57 \\
\hline Bottom-up (Relative MAFE) & 9) $\mathrm{TDI}+\mathrm{AR}$ & $0.96^{* *}$ & 0.98 & $0.93^{* *}$ & $0.96^{* * *}$ & 0.98 & 0.99 \\
\hline \multicolumn{8}{|l|}{ GDP } \\
\hline Direct (MAFE) & TDI & 0.41 & 0.62 & 0.31 & 0.49 & 0.49 & 0.71 \\
\hline Bottom-up with main aggregates (Relative MAFE) & 1)+3)+4)+6)+8) & $0.87^{* *}$ & 0.98 & 0.91 & 1.12 & $0.86^{*}$ & 0.91 \\
\hline Bottom-up with detailed components (Relative MAFE) & $2)+3)+5)+7)+9)$ & 0.95 & 1.07 & 1.01 & 1.32 & 0.92 & 0.94 \\
\hline
\end{tabular}

TABLE 8. MAFE results for the bottom-up approach 